\title{
Quantitative Description of Temperature Induced Self-Aggregation Thermograms Determined by Differential Scanning Calorimetry
}

\author{
Leonardo Chiappisi, ${ }^{\dagger}{ }^{\dagger}$ Giuseppe Lazzara, ${ }^{\ddagger}$ Michael Gradzielski, ${ }^{\dagger}$ and Stefana Milioto ${ }^{\ddagger}$ \\ ${ }^{\dagger}$ Stranski-Laboratorium für Physikalische Chemie und Theoretische Chemie, Institut für Chemie, Technische Universität Berlin, Sekr. \\ TC 7, Strasse des 17. Juni 124, D-10623 Berlin, Germany \\ ‡Dipartimento di Chimica "S. Cannizzaro", Universitá degli studi di Palermo, Viale delle Scienze, Parco D’Orleans, 90128 Palermo, \\ Italy
}

\section{Supporting Information}

ABSTRACT: A novel thermodynamic approach for the description of differential scanning calorimetry (DSC) experiments on self-aggregating systems is derived and presented. The method is based on a mass action model where temperature dependence of aggregation numbers is considered. The validity of the model was confirmed by describing the aggregation behavior of poly(ethylene oxide)poly(propylene oxide) block copolymers, which are well-known to exhibit a strong temperature dependence. The quantitative description of the thermograms could be performed without any discrepancy between calorimetric and van 't Hoff enthalpies, and moreover, the aggregation numbers obtained from the best fit of the DSC experiments are in good agreement with those obtained by light scattering experiments corroborating the assumptions done in the derivation of the new model.

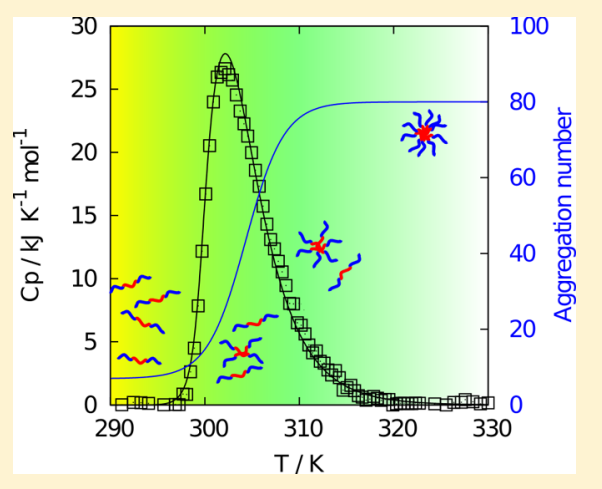

\section{INTRODUCTION}

The self-assembly of molecules in water to form aggregates has been intensively studied in the past decades, and nowadays, a number of well-established thermodynamic models for the description of such processes are known. The most studied selfaggregation process is the association of surfactants into micelles for which a huge amount of experimental data is reported. According to the pseudophase transition model, the most important parameter for the self-aggregation of surfactants is the critical micellar concentration (CMC). This quantity defines the threshold where surfactants start to form aggregates, and it is correlated to the standard free energy of micellization $\left(\Delta G_{\mathrm{m}}^{\circ}\right)$ :

$$
\Delta G_{\mathrm{m}}^{\circ}=R T \ln \mathrm{CMC}
$$

where $R$ and $T$ are the gas constant and the absolute temperature, respectively. The temperature at which the aggregation process takes place, for a given concentration, is defined as the critical micelle temperature (CMT), in analogy to the CMC. A number of techniques are available for the determination of both the CMC and CMT, from surface tension to scattering methods.

The direct determination of the enthalpy of micellization $\left(\Delta H_{\mathrm{m}}\right)$ is by far less immediate, as accurate calorimetric experiments are needed. ${ }^{1-3}$ Therefore, a common procedure exploits the temperature dependence of the CMC and its correlation to the enthalpy of micellization by means of the van't Hoff equation:

$$
\left(\frac{\partial \ln \mathrm{CMC}}{\partial \mathrm{T}}\right)_{P}=-\frac{\Delta H_{\mathrm{m}}^{\circ}}{R T^{2}}
$$

However, as A. Holtzer and M. Holtzer ${ }^{4}$ pointed out in 1974, when the aggregation number is not constant within the investigated temperature range, eq 2 does not always lead to reliable results and the following expression should be used: ${ }^{4}$

$$
\begin{aligned}
& R T^{2}\left(\frac{\partial \ln \mathrm{CMC}}{\partial T}\right)_{P} \\
& \quad=-\Delta H_{\mathrm{m}}^{\circ}(N)+T\left[\frac{\partial\left(G_{N+1}-G_{N}\right)}{\partial N}\right]_{T, P}\left(\frac{\partial N}{\partial T}\right)_{P}
\end{aligned}
$$

where $G_{N+1}$ and $G_{N}$ are the free energies of the surfactant molecules into the micelles with aggregation number of $N+1$ and $N$, respectively. However, it is difficult to evaluate a priori the magnitude of the last term at the right-hand side of eq 3 .

The effect of temperature on the aggregation number is particularly relevant in nonionic ethoxylated surfactants ${ }^{5-9}$ and poly(oxyethylene)-based block copolymers. ${ }^{1,2,10-12}$ Accordingly, for these systems strong discrepancies between van't Hoff and calorimetric enthalpy were evidenced. ${ }^{2,13,14}$ Such a discrepancy was either ascribed to the polydisperse nature of the used surfactant or justified by assuming a cooperative mechanism for the micelle formation.

Here, we report a new approach for the quantitative description of the differential scanning calorimetry (DSC) experiment on the aggregation process of amphiphilic molecules.

Received: September 7, 2012

Revised: November 17, 2012

Published: November 21, 2012 
Table 1. Characteristics of the Block Copolymers used in this Work

$\begin{array}{ccc}\text { abbreviation } & \text { structure formula }{ }^{a} & \text { molecular weight }^{a}\left[\mathrm{~g} \mathrm{~mol}{ }^{-1}\right] \\ \text { P65 } & \mathrm{EO}_{18}-\mathrm{PO}_{30}-\mathrm{EO}_{18} & 3400 \\ \text { P85 } & \mathrm{EO}_{26}-\mathrm{PO}_{40}-\mathrm{EO}_{26} & 4600 \\ \text { T904 } & \left(\mathrm{EO}_{15} \mathrm{PO}_{17}\right)_{2} \mathrm{NCH}_{2} \mathrm{CH}_{2} \mathrm{~N}\left(\mathrm{EO}_{15} \mathrm{PO}_{17}\right)_{2} / M_{\mathrm{n}} & 1.10^{b} \\ { }^{a} \text { According to BASF. EO and PO are ethylene oxide and propylene oxide units, respectively. }{ }^{b} \text { Ref } 18 .{ }^{c} \text { Ref } 19 .{ }^{c}{ }^{d} \text { Ref } 20 .^{c}\end{array}$

As a case study, we selected two different poly(oxyethylene)poly(oxypropylene)-poly(oxyethylene) block copolymers, Pluronic P65 $\left(\mathrm{EO}_{18}-\mathrm{PO}_{30}-\mathrm{EO}_{18}\right)$ and P85 $\left(\mathrm{EO}_{26}-\mathrm{PO}_{40}-\mathrm{EO}_{26}\right)$ with their aggregation behavior being well-known; moreover, they exhibit a large enthalpy of micellization and the aggregation behavior is strongly induced by temperature. ${ }^{15-17}$ Furthermore, the model has been also applied to a block copolymer with different geometry, namely, the star-block copolymer Tectronic T904 $\left(\left(\mathrm{EO}_{15} \mathrm{PO}_{17}\right)_{2} \mathrm{NCH}_{2} \mathrm{CH}_{2} \mathrm{~N}\left(\mathrm{EO}_{15} \mathrm{PO}_{17}\right)_{2}\right)$. To take into account eventual effects arising from the presence of a broad size distribution of the used polymers, we compare the results of the nonconstant aggregation number model with those obtained from a second model, where the polydispersity of the polymer is considered.

\section{MATERIALS AND METHODS}

The poly(oxyethylene)-poly(oxypropylene) based block copolymers were a kind gift of BASF, Germany, and their characteristics are summarized in Table 1. Solutions were prepared by mass using Milli-Q water. Before each measurement, the solutions were allowed to equilibrate for at least $48 \mathrm{~h}$.

Differential Scanning Calorimetry. Measurements were performed with a micro-DSC III 106 (Setaram, France). The stainless steel vessel $\left(1 \mathrm{~cm}^{3}\right)$ was filled with $\sim 500 \mathrm{mg}$ of solution and the reference vessel with the same amount of deionized water. The calibration was carried out using naphthalene. The baseline was subtracted according to ref 21. To ensure that the thermogram is recorded under thermodynamic equilibrium conditions, the heating rate was varied from 0.6 to $0.025^{\circ} \mathrm{C} \cdot \mathrm{min}^{-1}$. Figure 1 shows that the curves recorded at $0.1,0.05$, and $0.025{ }^{\circ} \mathrm{C} \cdot \mathrm{min}^{-1}$ perfectly overlap. The thermograms recorded at $0.1{ }^{\circ} \mathrm{C} \cdot \mathrm{min}^{-1}$ were used since they have the best signal-tonoise ratio.

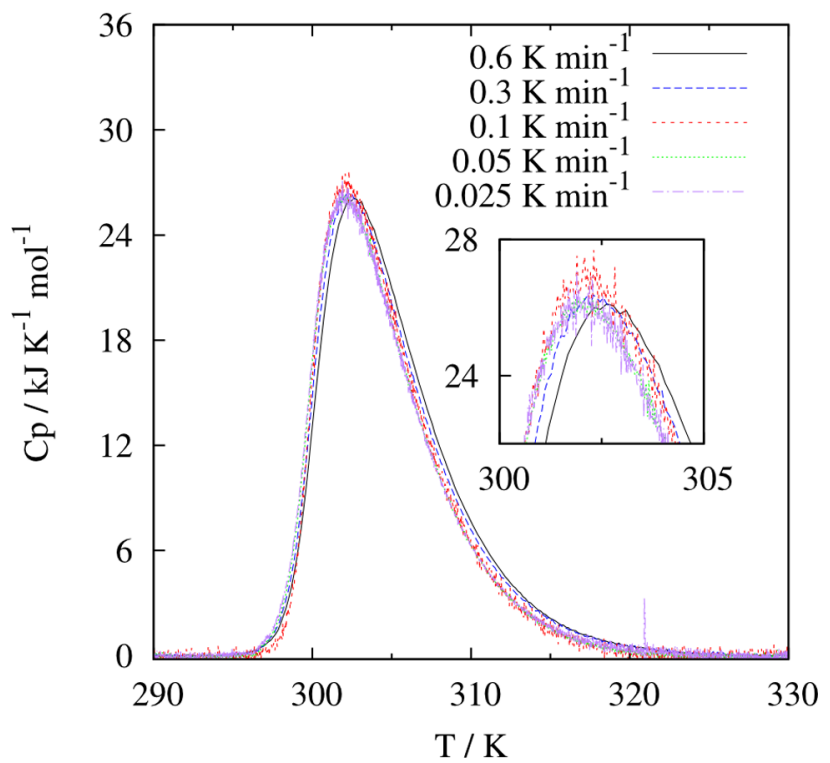

Figure 1. Apparent molar heat capacity of the aqueous P85 solution $\left(5 \%_{\mathrm{w} / \mathrm{w}}\right)$ as a function of temperature recorded at different heating rates.
Static Light Scattering (SLS). Experiments were performed on a compact ALV/CGS-3 instrument, equipped with a $\mathrm{He}-\mathrm{Ne}$ laser $(\lambda=$ $632.8 \mathrm{~nm}$ ). All measurements were carried out in a thermostatted toluene bath. The scattering intensity was recorded for different scattering vectors $(q)$

$$
q=\frac{4 \pi n}{\lambda} \sin (\theta / 2)
$$

where $n$ is the refractive index of the solution and $\theta$ the scattering angle, which was varied between $45^{\circ}$ and $150^{\circ}$. Due to isotropic scattering of the micelles within the recorded $q$-range, the intensity at $I(q \rightarrow 0)$ was obtained as average of the intensities recorded at the different angles. Calibration for absolute scale was done using tolouene as a reference. In order to determine the mean molecular weight of the aggregates, the forward scattering intensity from the polymer solution $I(0)$ has to be considered.

$$
I(0, T)=I(0, T)_{\text {tot }}-I(0)_{\text {solv }}
$$

where $I(0)_{\text {solv }}$ is the forward scattering intensity of the solvent and the cuvette. The mean weight average molecular weight of the aggregates $\left(M_{\mathrm{w}}\right)$ are determined as

$$
\frac{I(0, T)}{S(0, T)}=K_{\mathrm{L}} c M_{\mathrm{w}}
$$

where $c$ is the total concentration of the polymer. $S(0)$ is the scattering structure factor at $q \rightarrow 0$ and $K_{\mathrm{L}}$ is the optical constant, defined as follows:

$$
K_{\mathrm{L}}=\frac{4 \pi^{2}}{\lambda^{4} N_{\mathrm{A}}} n_{0}^{2}\left(\frac{\mathrm{d} n}{\mathrm{~d} c}\right)^{2}
$$

where $\mathrm{d} n / \mathrm{d} c$ is the refractive index increment, calculated as $\mathrm{d} n / \mathrm{d} c=$ $0.141-2.74 \times 10^{-4} T{ }^{22}$ The same $\mathrm{d} n / \mathrm{d} c$ for the micellized and free surfactant was assumed.

The scattering structure factor at $q \rightarrow 0$ can be approximated using the Carnahan-Starling equation of states: ${ }^{23}$

$$
S(0)=\frac{(1-\phi)^{4}}{\phi^{4}-4 \phi^{3}+(1+2 \phi)^{2}}
$$

where $\phi$ is the effective hard sphere volume fraction of the hydrated aggregate. For the calculations, water contents of $30 \%_{\mathrm{v} / \mathrm{v}}$ in the PPO core and $80 \%_{\mathrm{v} / \mathrm{v}}$ in the $\mathrm{PEO}$ corona were assumed. ${ }^{24,25}$

New Approach for DSC Data Analysis. For the description of the fraction of micellized surfactant, we make the following assumptions: The aggregation process is described by the closed-association model, where unimers and monodisperse micelles are at equilibrium

$$
N U \rightleftarrows M_{N}
$$

where $U$ is the unimeric surfactant and $M_{N}$ is a micelle formed by $\mathrm{N}$ molecules. The equilibrium constant is given by

$$
K_{\mathrm{eq}} \approx \frac{\left[M_{N}\right]}{[U]^{N}}
$$

where $\left[M_{N}\right]$ and $[U]$ are the mole fraction of the micelle and the unimers, respectively. The standard Gibbs free energy of micellization per mole of surfactant $\overline{\Delta G_{\mathrm{m}}^{\circ}}$ is given by

$$
\overline{\Delta G_{\mathrm{m}}^{\circ}}=\frac{\Delta G_{\mathrm{m}}}{N}=-\frac{R T}{N} \ln \left[M_{N}\right]+R T \ln [U]
$$


by considering the micelles as a pure phase, $\left[M_{N}\right]=1$, and therefore $\overline{\Delta G_{\mathrm{m}}^{\circ}}=R T \ln [U]$.

The aggregation number as a function of temperature, $N(T)$, is described by the following equation

$$
N(T)=N_{\min }+\left(N_{\min }-N_{\max }\right) /\left(1+10^{p\left(T-T_{0}\right)}\right)
$$

where $N_{\min }$ and $N_{\max }$ are the minimum and maximum aggregation number, respectively. $T_{0}$ represents the temperature of the inflection point of the curve and $p$ influences the steepness of the curve. This function is advantageous because it effectively describes a finite growth process, foreseeing a minimum aggregation number needed for the formation of an aggregate. In addition, the parameters $T_{0}$ and $p$ allow us to tune the growth behavior. The presence of artifacts arising from the arbitrary choice of this particular function could be excluded by comparing two thermograms which were simulated using similar aggregation numbers, but different equation describing them. More details can be found in the Appendix.

The change in the standard free energy and enthalpy of micellization per mole of surfactant, $\overline{\Delta G_{\mathrm{m}}^{\circ}}$ and $\overline{\Delta H_{\mathrm{m}}^{\circ}}$, respectively, is independent of the aggregation number.

The change in the heat capacity per mole of surfactant for the aggregation process, $\overline{\Delta \mathrm{Cp}_{\mathrm{m}}^{\circ}}$, is independent of the aggregation number and constant within the investigated temperature range.

The dependence of the standard free energy of micellization per mole of surfactant can be described by the Gibbs-Helmholtz equation:

$$
\begin{aligned}
\frac{\overline{\Delta G_{\mathrm{m}}^{\mathrm{o}}}(T)}{T}= & \frac{\overline{\Delta G_{\mathrm{m}}^{\mathrm{o}}}\left(T^{*}\right)}{T^{*}}+\left[\overline{\Delta H_{\mathrm{m}}^{\mathrm{o}}}\left(T^{*}\right)-T^{*} \overline{\Delta \mathrm{Cp}_{\mathrm{m}}^{\mathrm{o}}}\right]\left[\frac{1}{T}-\frac{1}{T^{*}}\right] \\
& -\overline{\Delta C \mathrm{p}_{\mathrm{m}}^{\mathrm{o}}} \ln \left(\frac{T}{T^{*}}\right)
\end{aligned}
$$

where $\overline{\Delta G_{\mathrm{m}}^{\mathrm{o}}}\left(T^{*}\right)$ and $\overline{\Delta H_{\mathrm{m}}^{\mathrm{o}}}\left(T^{*}\right)$ are the change of free energy and enthalpy of the process per mole of surfactant at the temperature $T^{*}$.

Note that the quantities $\overline{\Delta G_{\mathrm{m}}^{\mathrm{o}}}, \overline{\Delta H_{\mathrm{m}}^{\mathrm{o}}}$ and $\overline{\Delta \mathrm{Cp}_{\mathrm{m}}^{\mathrm{o}}}$ are related to the transferring of a monomer from the aqueous phase to the micellar aggregate.

The equilibrium constant for the micellization process (eq 9) can be rewritten as a function of $\overline{\Delta G_{\mathrm{m}}^{\mathrm{o}}}$ as

$$
K_{\text {eq }}(T)=\mathrm{e}^{-\left[N(T) \overline{\Delta G_{\mathrm{m}}^{\circ}}(T) / R T\right]}
$$

By combining eqs 13 and 14 , one obtains

$$
\begin{aligned}
K_{\mathrm{eq}}(T)= & \exp \left\{-\left[\frac { N ( T ) } { R } \left[\frac{\overline{\Delta G_{\mathrm{m}}^{\mathrm{o}}}\left(T^{*}\right)}{T^{*}}+\left(\overline{\Delta H_{\mathrm{m}}^{\mathrm{o}}}\left(T^{*}\right)-T^{*} \overline{\Delta C \mathrm{p}_{\mathrm{m}}^{\mathrm{o}}}\right)\right.\right.\right. \\
& \left.\left.\left.\left(\frac{1}{T}-\frac{1}{T^{*}}\right)-\overline{\Delta \mathrm{C \textrm {p } _ { \mathrm { m } } ^ { \mathrm { o } }}} \ln \left(\frac{T}{T^{*}}\right)\right]\right]\right\}
\end{aligned}
$$

The fraction of nonaggregated surfactant $\chi$ is given by:

$$
\chi=\frac{[\mathrm{U}]}{c}
$$

where $c=N\left[\mathrm{M}_{N}\right]+[\mathrm{U}]$ is the total concentration of surfactant. The amount of unimeric surfactant can finally be described as a function of temperature by combining eqs 10,15 , and 16 as

$$
\begin{aligned}
\frac{1-\chi(T)}{\chi(T)^{N(T)}}= & N(T) \cdot c^{N(T)-1} . \\
& \exp \left\{-\left[\frac { N ( T ) } { R } \left[\frac{\overline{\Delta G_{\mathrm{m}}^{\mathrm{o}}}\left(T^{*}\right)}{T^{*}}+\left(\overline{\Delta H_{\mathrm{m}}^{\mathrm{o}}}\left(T^{*}\right)\right.\right.\right.\right. \\
& \left.\left.\left.\left.-T^{*} \overline{\Delta C \mathrm{p}_{\mathrm{m}}^{\mathrm{o}}}\right)\left(\frac{1}{T}-\frac{1}{T^{*}}\right)-\overline{\Delta \mathrm{Cp}_{\mathrm{m}}^{\mathrm{o}}} \ln \left(\frac{T}{T^{*}}\right)\right]\right]\right\}
\end{aligned}
$$

A quantitative analysis of the DSC thermogram could be obtained by multiplying the heat of micellization with the fraction of surfactant which is aggregating upon temperature changing

$$
\mathrm{Cp}(T)=-\frac{\partial \chi(T)}{\partial T}\left[\overline{\Delta H_{\mathrm{m}}^{\mathrm{o}}}\left(T^{*}\right)+\overline{\Delta \mathrm{Cp}_{\mathrm{m}}^{\mathrm{o}}}\left(T-T^{*}\right)\right]
$$

An analytical expression for the quantitative description of the micellization peak is given in the Appendix. The fits were done with the Sasfit software. ${ }^{26}$ For the fitting procedure, eq 17 was solved using a robust Newton-Raphson root finding algorithm. The plugin can be obtained upon request.

\section{RESULTS AND DISCUSSION}

Varying Aggregation Number - Shape of the Thermograms. In Figure 2, four simulated thermograms, which only

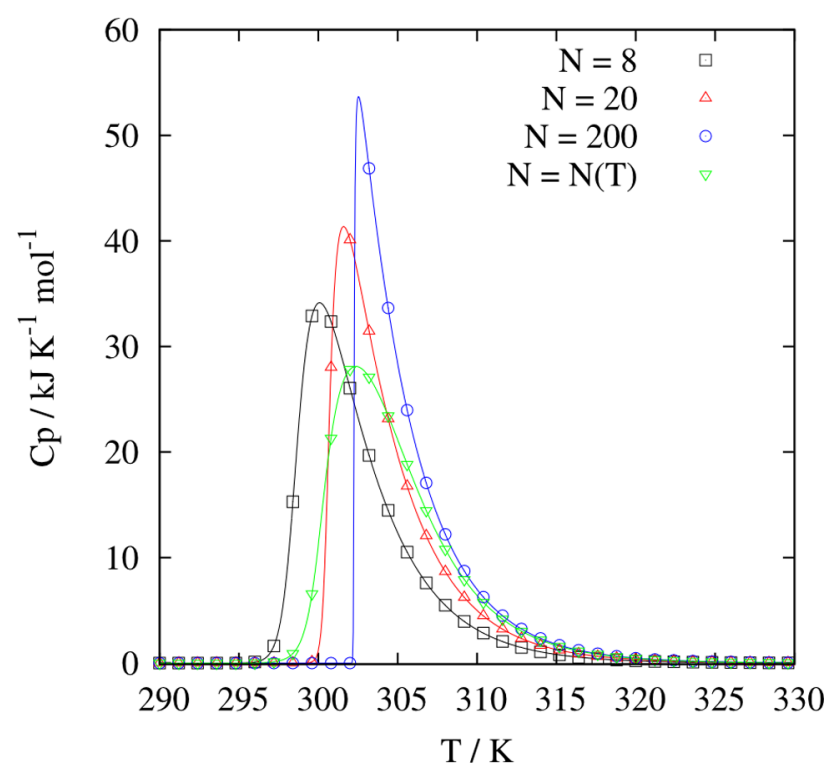

Figure 2. Simulated thermograms for a surfactant solution, with $\overline{\Delta G_{\mathrm{m}}^{\mathrm{o}}}=$ $21.68 \mathrm{~kJ} \mathrm{~mol}^{-1}, \overline{\Delta H_{\mathrm{m}}^{\mathrm{o}}}=135 \mathrm{~kJ} \mathrm{~mol}^{-1}, \mathrm{CMT}=302.5 \mathrm{~K}$, and molality of $0.01 \mathrm{~mol} \mathrm{~kg}^{-1}$. The variation of the aggregation number is described by eq 12 with $N_{\min }=8, N_{\max }=80, T_{0}=305 \mathrm{~K}$, and $p=-0.21$.

differ in the aggregation number, are shown. In all cases, the same changes in enthalpy and free energy, CMT, and concentration were set. In order to focus only on the effect of aggregation number, the temperature dependence of the enthalpy has been neglected. One can immediately notice that the aggregation processes which are characterized by smaller aggregation numbers show a less steep onset than for a typical phasetransition process (for which $N \rightarrow \infty$ ). The second factor is that for low $N$ neither the peak onset nor the peak maximum correspond to the CMT, since that process starts at lower temperatures. When an increasing aggregation number is assumed, a smooth onset, typical for small $N$, is observed. On the contrary, the shape of the micellization peak at high temperatures is ruled by high aggregation numbers, as proven by the fact that the curve perfectly overlaps with that calculated for $N=200$.

Varying Aggregation Number - Experimental Results on Some Common Surfactants. The CMT, $\overline{\Delta G_{\mathrm{m}}^{\mathrm{o}}}, \overline{\Delta H_{\mathrm{m}}^{\mathrm{o}}}$ and $\overline{\Delta \mathrm{Cp}_{\mathrm{m}}^{\mathrm{o}}}$ values for the examined surfactants are reported in Table 
Table 2. Concentration, CMT, $\overline{\Delta G_{\mathrm{m}}^{\mathrm{o}}}$ and $\overline{\Delta H_{\mathrm{m}}^{\mathrm{o}}}$ at the CMT, $\overline{\Delta \mathrm{Cp}_{\mathrm{m}}^{\mathrm{o}}}$ Values, and Fitting Parameters Determining the Aggregation Number for the Different Examined Polymer Solutions ${ }^{a}$

\begin{tabular}{|c|c|c|c|c|c|c|c|c|c|}
\hline & $c\left[\mathrm{~mol} \mathrm{~kg}^{-1}\right]$ & CMT $[\mathrm{K}]$ & $\overline{\Delta G_{\mathrm{m}}^{\mathrm{o}}}(\mathrm{CMT})\left[\mathrm{kJ} \mathrm{mol}^{-1}\right]$ & $\overline{\Delta H_{\mathrm{m}}^{\mathrm{o}}}(\mathrm{CMT})\left[\mathrm{kJ} \mathrm{mol}^{-1}\right]$ & $\overline{\Delta \mathrm{CP}_{\mathrm{m}}^{\mathrm{o}}}\left[\mathrm{kJ} \mathrm{mol}{ }^{-1} \mathrm{~K}^{-1}\right]$ & $N_{\min }$ & $N_{\max }$ & $\mathrm{p}$ & $T_{0}[\mathrm{~K}]$ \\
\hline P65 & 0.0083 & 311.8 & -22.83 & $134(4.5)$ & $0.4(0.01)$ & 9.7 & 50.5 & -0.184 & 311.5 \\
\hline P65 & 0.0143 & 309.5 & -21.27 & $144(4.8)$ & $-2.7(-0.09)$ & 11.4 & 72.7 & -0.21 & 310.9 \\
\hline P65 & 0.0311 & 306.9 & -19.11 & $151(5.0)$ & $-2.1(-0.07)$ & 6.77 & 67.9 & -0.156 & 310.3 \\
\hline P85 & 0.055 & 303.9 & -23.30 & $223(5.6)$ & $-5.3(-0.13)$ & 5.76 & 78.6 & -0.206 & 307.2 \\
\hline P85 & 0.0107 & 302.3 & -21.50 & $228(5.8)$ & $-5.6(-0.14)$ & 6.5 & 81.6 & -0.209 & 306.6 \\
\hline P85 & 0.0244 & 298.7 & -19.21 & $206(5.2)$ & $-0.4(-0.01)$ & 4.9 & 77.9 & -0.177 & 303.3 \\
\hline T904 & 0.0084 & 301.8 & -22.06 & $260(3.8)$ & $-9.3(-0.14)$ & 9.8 & 79.08 & -0.355 & 301.8 \\
\hline
\end{tabular}

The best fit curves shown afterward were obtained optimizing only the parameters governing the aggregation number, i.e., $N_{\min }$ $N_{\max } p$, and $T_{0}$ of eq 12 . These parameters are also shown in Table 2. $\overline{\Delta \mathrm{Cp}_{\mathrm{m}}^{\mathrm{o}}}$ was obtained from the difference in baseline before and after the peak. The CMT was determined from the peak maximum, the enthalpy of micellization $\left(\overline{\Delta H_{\mathrm{m}}^{\mathrm{o}}}\right)$ from the area of the micellization peak. The $\overline{\Delta G_{\mathrm{m}}^{\mathrm{o}}}$ at the CMT was calculated using eq 1 , with the CMC being the total polymer concentration.

In Figure 3, the thermogram of the $5 \%_{w / w}$ P85 aqueous solution is given. In the same plot, the aggregation numbers

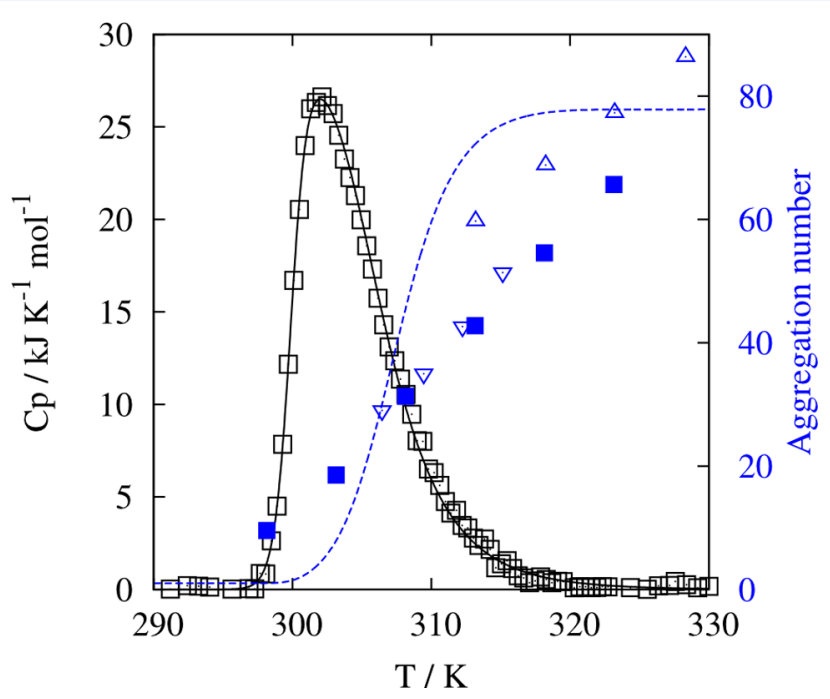

Figure 3. Apparent molar heat capacity of the $5 \%{ }_{\mathrm{w} / \mathrm{w}}\left(0.0102 \mathrm{~mol} \mathrm{~kg}^{-1}\right)$ P85 aqueous solution (empty squares) as a function of temperature. The solid black line represents best fit of heat capacity data according to eq 18. The broken blue line represents mean aggregation number according to eq 19. Blue squares are $N_{\text {agg }}$ determined by SLS; blue $\Delta$ and blue $\nabla$ are $N_{\text {agg }}$ from ref 27 (SANS at $1 \%_{\mathrm{w} / \mathrm{w}}$ ) and ref 11 (SANS at $\left.10 \%_{\mathrm{w} / \mathrm{w}}\right)$, respectively.

obtained by light scattering experiments performed on the same solution and those from the literature are reported. In order to best compare the results with the light scattering data, the mean aggregation numbers, calculated as the average between the molecular weight of the aggregates and unimers:

$$
\bar{N}(T)=\chi+(1-\chi)\left(N_{\min }+\frac{N_{\min }-N_{\max }}{1+10^{p\left(T-T_{0}\right)}}\right)
$$

is also shown. The literature data were obtained by small angle neutron scattering (SANS) at concentrations between $1 \%$ and
$10 \%_{\mathrm{w} / \mathrm{w}^{*}}{ }^{11,27}$ Thermograms and aggregation numbers of the $5 \%_{w / w}$ aqueous solution of P65 and T904 are reported in Figure 4

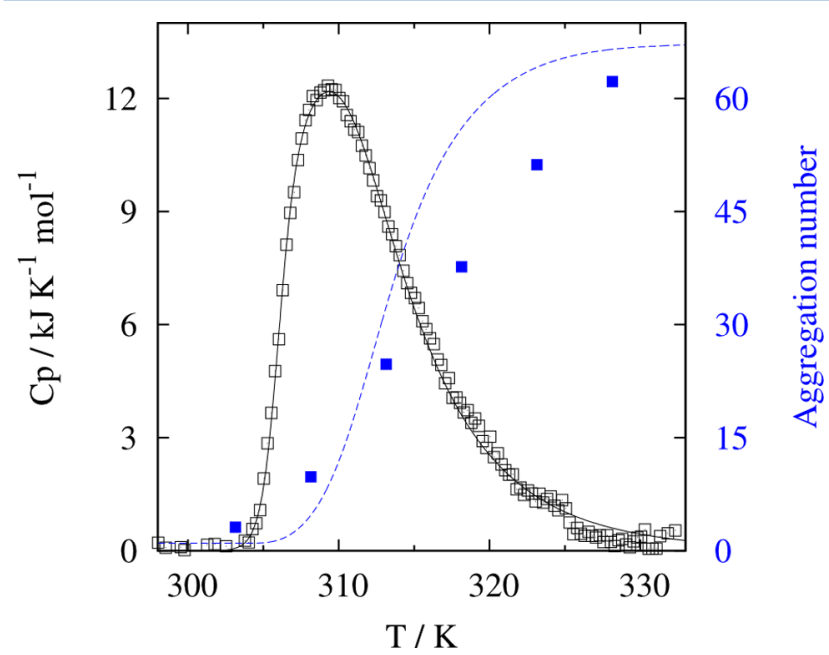

Figure 4. Apparent heat capacity of a $5 \%_{w / w}\left(0.0143 \mathrm{~mol} \mathrm{~kg}^{-1}\right)$ P65 aqueous solution (black empty squares) as a function of temperature. The solid black line represents best fit of the heat capacity data. The broken blue line represents mean aggregation number according to eq 19. Blue squares are $N_{\text {agg }}$ determined by SLS.

and Figure 5, respectively. Thermograms and best fitting curves of P85 and P65 solution at 2.5, 5.0, and $10 \%_{\mathrm{w} / \mathrm{w}}$ are shown in the Supporting Information. The model is able to describe all the key features of the curve, including the aggregation numbers, which are in a satisfactory agreement with those obtained in the present paper and with other methods. In all cases, the initial size of the micelles is rather small if compared with other surfactants and is found to be between five and ten polymers per micelles. It is interesting to note that $T_{0}$ follows the same trend of the CMT, indicating that the temperature at which most of the polymer chains aggregate roughly coincides with the one of maximal growth of the micelles.

Effect of Polymer Inhomogeneities. The surfactants used are all of technical grade and are polydisperse. Beside the distribution in the length of the blocks, diblocks and single PPO blocks are also present. The polydispersity was shown to influence the aggregation behavior and, therefore, the thermograms of the studied block copolymers. ${ }^{28}$ Batsberg et al. fractionated the P85 block copolymer and presented the thermograms of three different fractions showing that depending on the hydrophobicity of the fraction both shape and position of the micellization peak are changed. ${ }^{28}$ Oppositely, Paterson et al. claim that the presence of hydrophobic moieties has an effect on 


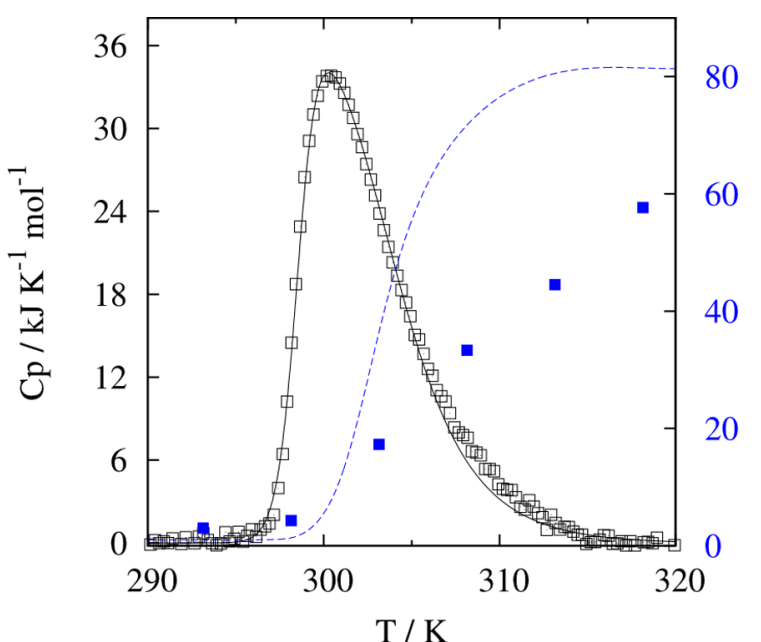

Figure 5. Apparent heat capacity of a $5 \%_{w / w}\left(0.0084 \mathrm{~mol} \mathrm{~kg}^{-1}\right)$ T904 aqueous solution (empty black squares) as a function of temperature. The solid black line represents best fit of the heat capacity data. The broken blue line represents mean aggregation number according to eq 19. Blue squares are $N_{\text {agg }}$ determined by SLS.

the CMT, but not on the overall aggregation behavior. ${ }^{13} \mathrm{~A}$ micellization process, where only molecules with similar block length aggregate, would be kinetically unfavored, since it takes a rather long time until enough molecules with the "correct" architecture come together. Accordingly, it is more likely that the most hydrophobic molecules start the aggregation process, but they aggregate with their neighbors rather than with compounds of similar hydrophobicity.

In order to consider the effect of the polydispersity of the polymer, a simulated thermogram assuming a Schultz-Zimm distribution of the PO units was obtained as

$$
\Delta \mathrm{Cp}_{\mathrm{tot}}(T)=\int_{0}^{\infty} z s\left(N_{\mathrm{PO}}\right) \Delta \mathrm{Cp}\left(T, N_{\mathrm{PO}}\right) \mathrm{d} N_{\mathrm{PO}}
$$

where $z s\left(N_{\mathrm{PO}}\right)$ is the weighing factor for a thermogram $\Delta \mathrm{Cp}(T$, $N_{\text {PO }}$ ) arising from a monodisperse polymer with $N_{\text {PO }}$ propylenoxide units given by

$$
z s\left(N_{\mathrm{PO}}\right)=\frac{k^{k}}{\Gamma(k) \overline{N_{\mathrm{PO}}}}\left(\frac{N_{\mathrm{PO}}}{\overline{N_{\mathrm{PO}}}}\right)^{k-1} \exp \left(-k \frac{N_{\mathrm{PO}}}{\overline{N_{\mathrm{PO}}}}\right)
$$

with $\overline{N_{\mathrm{PO}}}$ being the average number of $\mathrm{PO}$ units per surfactant molecule. The dependence of the change in the standard free energy and enthalpy from the number of propylenoxide units was already described and the following values were used: ${ }^{29}$

$$
\begin{aligned}
& \overline{\Delta G_{\mathrm{m}}^{\mathrm{o}}} / \mathrm{kJ} \mathrm{mol}^{-1}=-5.05-0.15 N_{\mathrm{PO}} \\
& \overline{\Delta H_{\mathrm{m}}^{\mathrm{o}}} / \mathrm{kJ} \mathrm{mol}^{-1}=76.7+3.25 N_{\mathrm{PO}}
\end{aligned}
$$

In Figure 6, the simulated thermogram assuming a pseudophase transition model is compared with a simulated thermogram obtained for a monodisperse surfactant with the same $\overline{\Delta G_{\mathrm{m}}^{\mathrm{o}}}$ and $\overline{\Delta H_{\mathrm{m}}^{\mathrm{o}}}$ values. For the sake of completeness, the experimentally determined thermogram for a $5 \%_{\mathrm{w} / \mathrm{w}}$ solution of P85 is reported in the same plot. Despite the model being very simple, where cooperativity between polymers with different $\mathrm{PO}$ units is neglected, some of the features of the micellization peak can be reproduced, such as the smoothing of the onset, and it is likely that the shape of experimentally obtained thermograms of such systems is influenced by the change in aggregation number

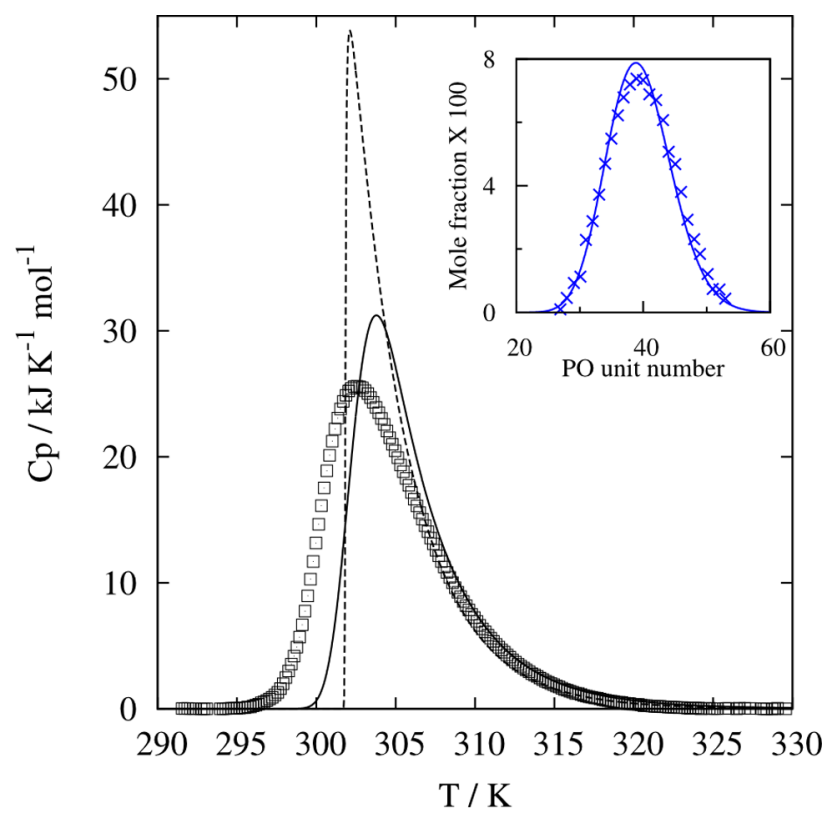

Figure 6. Apparent molar heat capacity of the $5 \%_{\mathrm{w} / \mathrm{w}}$ aqueous solution of P85 (empty squares) as a function of temperature. Broken line represents simulated curve using the pseudophase transition model for a monodisperse surfactant system, solid line the simulated curves using the pseudophase transition model for a polydisperse surfactant. In the inset, the PO unit distribution (crosses) taken from ref 28 and the one used for the calculation (full line) are reported, obtained using eq 21 with $k=60$ and $\overline{N_{\mathrm{PO}}}=39.5$.

as well as by the presence of a rather broad distribution of molecules with different tendency to aggregate.

Effect of Polydispersity of Micelle Aggregation Number. The aggregation number of the PEO-PPO-PEO block copolymer micelles was shown to be slightly polydisperse, with standard deviation in sizes on the order of $\sigma=0.1-0.2$, depending on the experimental conditions. ${ }^{11,12,24}$ This effect has not been considered until now and will be discussed in the following lines.

The aggregation numbers can be regarded as normally distributed around an optimum, and the formation of micelles with different $N_{\text {agg }}$ can be considered an independent process. Accordingly, thermograms of the micellization process with polydisperse aggregation numbers can be described as

$$
\Delta \mathrm{Cp}_{\text {tot }}(T)=\int_{0}^{\infty} g s\left(N_{\text {agg }}, T\right) \Delta \mathrm{Cp}\left(T, N_{\text {agg }}\right) \mathrm{d} N_{\text {agg }}
$$

where $g s\left(N_{\mathrm{agg}}, T\right)$ is the weighting factor for a thermogram $\Delta \mathrm{Cp}\left(T, N_{\mathrm{agg}}\right)$ arising from the formation of an aggregate of $N_{\text {agg }}(T)$ copolymer chains given by

$$
g s\left(N_{\mathrm{agg}}, T\right)=\frac{1}{\sigma \sqrt{2 \pi}} \exp \left(-\frac{\left[N_{\mathrm{agg}}(T)-\overline{N_{\mathrm{agg}}}(T)\right]^{2}}{2 \sigma^{2}}\right)
$$

with $\overline{N_{\text {agg }}}(T)$ being the average aggregation number at the temperature $T$. In Figure 7, the simulated thermograms arising from monodisperse and polydisperse aggregate formation are reported. The effect of rather large polydispersity influences mainly the initial part of the peak and the peak hight, not the maximum. The broadening of the onset can be ascribed to the presence of smaller aggregates, which, as demonstrated before, start to form at lower temperature. 


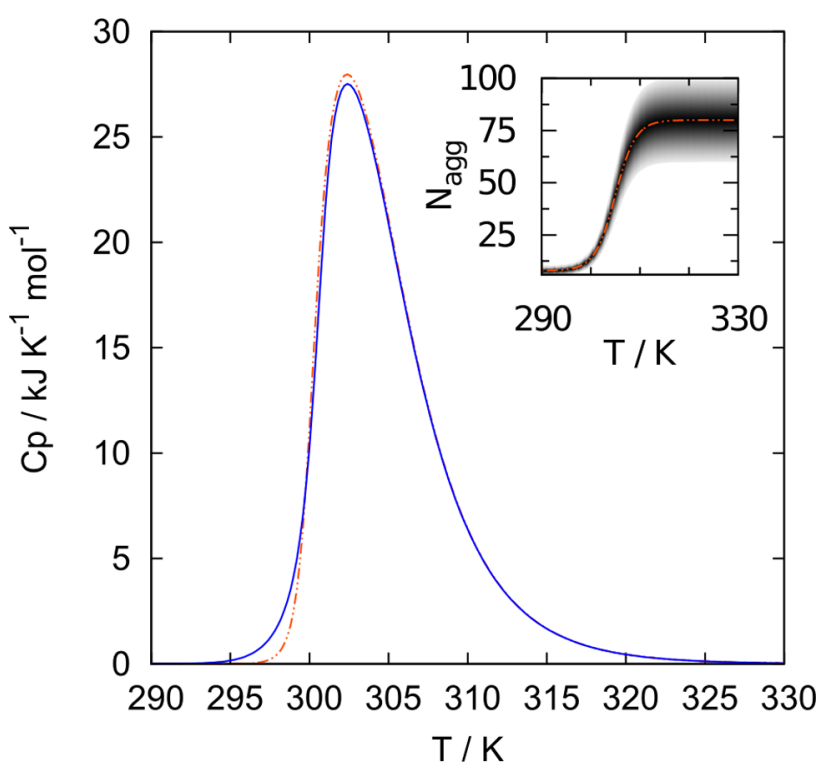

Figure 7. Simulated thermograms for a surfactant solution, with $\overline{\Delta G_{\mathrm{m}}^{\mathrm{o}}}=$ $-11.4 \mathrm{~kJ} \mathrm{~mol}^{-1}, \overline{\Delta H_{\mathrm{m}}^{\mathrm{o}}}=216 \mathrm{~kJ} \mathrm{~mol}^{-1}, \mathrm{CMT}=302.5 \mathrm{~K}$, and molality of $0.01 \mathrm{~mol} \mathrm{~kg}^{-1}$. The broken red line was calculated using aggregation numbers described by eq 12 with $N_{\min }=8, N_{\max }=80, T_{0}=305 \mathrm{~K}$, and $p=$ -0.21 . The solid blue lines were calculated with the same aggregation numbers but considering a standard deviation of $\sigma=0.2$. Inset represents mean aggregation numbers and their distribution as a function of temperature.

\section{CONCLUSIONS}

We reported a new thermodynamic model for the description of the temperature induced self-aggregation of amphiphilic molecules. The new model was employed to simulate the effect of different aggregation numbers on the output of differential scanning calorimetric experiments. In order to validate the model, a quantitative description of the apparent molar heat capacity of three different poly(oxypropylene)-poly(oxyethylene) block copolymer aqueous solutions was performed. The analysis of the DSC output could allow us to obtain aggregation numbers in a good agreement with those from static light scattering, thus confirming the validity of the model.

In order to evaluate possible effects arising from not having pure compounds as surfactants, a very simple model was used and a DSC thermogram for a polydisperse surfactant simulated, showing that not only low aggregation numbers, but also the presence of more hydrophobic molecules cause a broadening of the aggregation curve.

Furthermore, the effect of the formation of polydisperse micelles was also investigated. A DSC thermogram was simulated assuming a rather high polydispersity $(\sigma=0.2)$ in micellar aggregation number. A broadening of the peak at the onset and a slight shift of the peak maximum can be observed, while almost no changes at higher temperature can be seen. It is generally true that the shape of the calculated curves is highly sensitive to the minimum aggregation number $N_{\text {min }}$, whereas even big changes in the behavior of $N(T)$ at $T>C M T$ have a rather small effect on the calculated curve. This arises from the fact that for increasingly large $N$ one approaches the thermodynamic limit and is less and less sensitive to $N$, which mplies that reliable aggregation numbers are obtained only for the first part of the micellization process. Increased reliability can be achieved by fixing as many parameters as possible.
A number of works dealing with PEO-PPO-PEO block copolymers are available in the literature, most of them reporting and interpreting DSC curves. It is not the goal of the work to answer the question of whether the shape of the mizellization peak is due to increasing aggregation number rather than to the presence of a broad distribution of block sizes. Still, there is enough experimental evidence that both factors influence the shape of the curve. However, even if not explicitly considered, information on the polydispersity of the sample is contained in the nonconstant aggregation number. With increasing temperature, the most hydrophobic molecules will first dehydrate and form micellar aggregates. Upon increasing temperature, more and more surfactant molecules will dehydrate and probably will be encapsulated into already existing aggregates.

If P85 is considered, with the PO unit distribution reported in the literature ${ }^{28}$ and shown in Figure 6, assuming the 5\% most hydrophobic molecules form aggregates with $N_{\text {agg }}=6$, after all the surfactant molecules aggregated into existing micelles, the aggregation number rises to ca. 120. This simple calculation straightforwardly shows that polydispersity and variable aggregation numbers are strictly connected and that our model can also be employed for the description of polydispersed samples.

Finally, since the model is not linked to the shape or size of the aggregates, but only to the number of subunits forming them, it can in principle be applied to all temperature-induced phenomena, from micelle formation to polymer collapse, and so forth.

\section{APPENDIX}

\section{Effect of the choice of $N(T)$}

In this work, a sigmoid has been chosen for describing the dependence of the aggregation numbers from the temperature. This choice is arbitrary and was motivated above. In order to exclude particular effects arising from the choice of this function, almost the same aggregation numbers are described with two different functions, eq 12 and

$$
N^{\prime}(T)=N_{1}+N_{2} \operatorname{erf}\left(a\left(T-T_{0}\right)\right)
$$

The simulated micellization peaks are reported in Figure 8, and the small differences can be ascribed to the small differences in $N(T)$ and its derivatives, confirming that the function itself is not influencing the simulated curves.

\section{Analytical Expression for the Micellization Peak}

An analytical expression for the derivative of the fraction of micellized surfactant can be obtained by differentiating and rearranging both sides of eq 17

$$
-\frac{\partial \chi}{\partial T}=\chi^{n} \frac{\frac{\partial f(T)}{\partial T}+\frac{\partial N(T)}{\partial T} f(T) \log \chi}{1+N(T) f(T) \chi^{N(T)-1}}
$$

with

$$
\begin{aligned}
f(T)= & N(T) c^{N(T)-1} \\
& \exp \left\{-\left[\frac { N ( T ) } { R } \left[\frac{\overline{\Delta G_{\mathrm{m}}^{\mathrm{o}}}\left(T^{*}\right)}{T^{*}}+\left(\overline{\Delta H_{\mathrm{m}}^{\mathrm{o}}}\left(T^{*}\right)\right.\right.\right.\right. \\
& \left.\left.\left.\left.-T^{*} \overline{\Delta \mathrm{Cp}_{\mathrm{m}}^{\mathrm{o}}}\right)\left(\frac{1}{T}-\frac{1}{T^{*}}\right)-\overline{\Delta \mathrm{Cp}_{\mathrm{m}}^{\mathrm{o}}} \ln \left(\frac{T}{T^{*}}\right)\right]\right]\right\}
\end{aligned}
$$




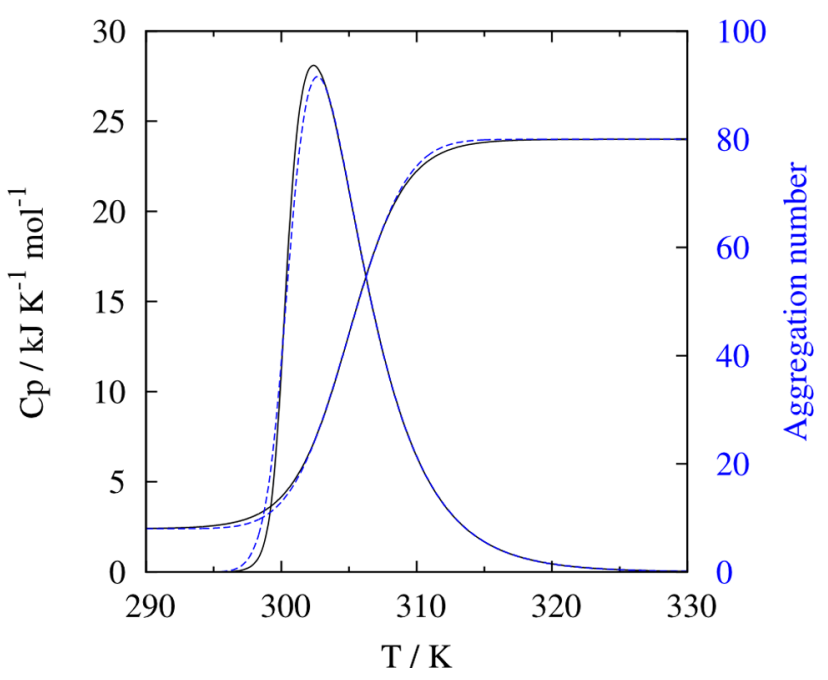

Figure 8. Simulated thermograms for a surfactant solution, with $\overline{\Delta G_{\mathrm{m}}^{\mathrm{o}}}=$ $-11.4 \mathrm{~kJ} \mathrm{~mol}^{-1}, \overline{\Delta H_{\mathrm{m}}^{\mathrm{o}}}=135 \mathrm{~kJ} \mathrm{~mol}^{-1}, \mathrm{CMT}=302.5 \mathrm{~K}$, and molality of $0.01 \mathrm{~mol} \mathrm{~kg}^{-1}$. The variation of the aggregation number is described by eq 12 with $N_{\min }=8, N_{\max }=80, T_{0}=305 \mathrm{~K}$, and $p=-0.21$ (full black line) or by 25 with $N_{1}=44, N_{2}=36, a=0.21$, and $T_{0}=305 \mathrm{~K}$ (blue broken line).

As shown before, $N(T)$ is of arbitrary choice and each function with continuous first derivative can be used.

\section{ASSOCIATED CONTENT}

\section{S Supporting Information}

Experimental thermograms of P65 and P85 2.5, 5.0 and $10.0 \%_{w / w}$ solutions with best fitting curves and aggregation numbers as determined by light scattering. This material is available free of charge via the Internet at http://pubs.acs.org.

\section{AUTHOR INFORMATION}

\section{Corresponding Author}

*E-mail: leonardo.chiappisi@tu-berlin.de.

\section{Notes}

The authors declare no competing financial interest.

\section{REFERENCES}

(1) Kadam, Y.; Singh, K.; Marangoni, D. G.; Ma, J. H.; Aswal, V. K.; Bahadur, P. Thermodynamic of micelle formation of nonlinear block copolymer Tetronic $®$ T904 in aqueous salt solution. Colloids Surf., A 2010, $369,121-127$

(2) Taboada, P.; Mosquera, V.; Attwood, D.; Yang, Z.; Booth, C. Enthalpy of micellisation of a diblock copoly(oxyethylene/oxypropylene) by isothermal titration calorimetry. Comparison with the van't Hoff value. Phys. Chem. Chem. Phys. 2003, 5, 2625-2627.

(3) Tiné, M. R.; Alderighi, M.; Duce, C.; Ghezzi, L.; Solaro, R. Effect of temperature on self-assembly of an ionic tetrapeptide. J. Therm. Anal. Calorim. 2011, 103, 75-80.

(4) Holtzer, A.; Holtzer, M. F. Use of the van't Hoff relation in determination of the enthalpy of micelle formation. J. Phys. Chem. 1974, $78,1442-1443$.

(5) Borbély, S. Aggregate structure in aqueous solutions of brij-35 nonionic surfactant studied by small-angle neutron scattering. Langmuir 2000, 16, 5540-5545.

(6) Bernheim-Groswasser, A.; Wachtel, E.; Talmon, Y. Micellar growth, network formation, and criticality in aqueous solutions of the nonionic surfactant $\mathrm{C}_{12} \mathrm{E}_{5}$. Langmuir 2000, 16, 4131-4140.

(7) Grell, E.; Lewitzki, E.; Schneider, R. Phase transitions in non-ionic detergent micelles. J. Therm. Anal. Calorim. 2002, 68, 469-478.
(8) Heerklotz, H.; Tsamaloukas, A.; Kita-Tokarczyk, K.; Strunz, P.; Gutberlet, T. Structural, volumetric, and thermodynamic characterization of a micellar sphere-to-rod transition. J. Am. Chem. Soc. 2004, 126, 16544-16552.

(9) Dong, R.; Hao, J. Complex fluids of poly(oxyethylene) monoalkyl ether nonionic surfactants. Chem. Rev. 2010, 110, 4978-5022.

(10) Goldmints, I.; Yu, G.; Booth, C.; Smith, K. A.; Hatton, T. A. Structure of (deuterated PEO)-(PPO)-(Deuterated PEO) block copolymer micelles as determined by small angle neutron scattering. Langmuir 1999, 15, 1651-1656.

(11) Hammouda, B. SANS from Pluronic P85 in d-water. Eur. Polym. J. 2010, 46, 2275-2281.

(12) Manet, S.; Lecchi, A.; Impéror-Clerc, M.; Zholobenko, V.; Durand, D.; Oliveira, C. L. P.; Pedersen, J. S.; Grillo, I.; Meneau, F.; Rochas, C. Structure of micelles of a nonionic block copolymer determined by SANS and SAXS. J. Phys. Chem. B 2011, 115, 1131811329.

(13) Paterson, I.; Armstrong, J.; Chowdhry, B. Z.; Leharne, S. Thermodynamic model fitting of the calorimetric output obtained for aqueous solutions of oxyethylene-oxypropylene-oxyethylene triblock copolymers. Langmuir 1997, 13, 2219-2226.

(14) Nixon, S. K.; Hvidt, S.; Booth, C. Micellization of block copolymer P94 in aqueous solution. J. Colloid Interface Sci. 2004, 1, 219223.

(15) Alexandridis, P.; Holzwarth, J. F.; Hatton, T. A. Micellization of poly(ethylene oxide)-poly(propylene oxide)-poly(ethylene oxide) triblock copolymers in aqueous solutions: thermodynamics of copolymer association. Macromolecules 1994, 9, 2414-2425.

(16) Alexandridis, P.; Hatton, T. A. Poly(ethylene oxide)-poly(propylene oxide)-poly(ethylene oxide) block-copolymer surfactants in aqueous-solutions and at interfaces - thermodynamics, structure, dynamics, and modeling. Colloids Surf., A 1995, 96, 1-46.

(17) Alexandridis, P.; Holzwarth, J. F. Differential scanning calorimetry investigation of the effect of salts on aqueous solution properties of an amphiphilic block copolymer (poloxamer). Langmuir 1997, 13, 60746082.

(18) Petrov, P.; Tsvetanov, C. B.; Jérôme, R. J. Phys. Chem. B 2009, 113, $7527-7533$

(19) Shar, J. A.; Obey, T. M.; Cosgrove, T. Adsorption studies of polyethers Part 1. Adsorption onto hydrophobic surfaces. Colloids Surf., A 1998, 136, 21-33.

(20) Al-Hanbali, O.; Onwuzo, N. M.; Rutt, K. J.; Dadswell, C. M.; Moghimi, S. M.; Hunter, A. C. Modification of the Stewart biphasic colorimetric assay for stable and accurate quantitative determination of Pluronic and Tetronic block copolymers for application in biological systems. Anal. Biochem. 2007, 361, 287-93.

(21) Malakhov, D. V.; Abou Khatwa, M. K. Constructing a selfconsistent integral baseline by using cubic splines. J. Therm. Anal. Calorim. 2007, 87, 595-599.

(22) Liu, Y.; Chen, S.; Huang, J. S. Light-scattering studies of concentrated copolymer micellar solutions. Macromolecules 1998, 31, $6226-6233$.

(23) Carnahan, N. F.; Starling, K. E. Equation of state for nonattracting rigid spheres. J. Chem. Phys. 1969, 51, 635-636.

(24) Pedersen, J. S.; Gerstenberg, M. C. The structure of P85 Pluronic block copolymer micelles determined by small-angle neutron scattering. Colloids Surf., A 2003, 213, 175-187.

(25) Yang, L.; Alexandridis, P.; Steytler, D. C.; Kositza, M. J.; Holzwarth, J. F. Small-angle neutron scattering investigation of the temperature-dependent aggregation behavior of the block copolymer Pluronic L64 in aqueous solution. Langmuir 2000, 16, 8555-8561.

(26) Kohlbrecher, J.; Bressler, I. Software package SASfit for fitting small-angle scattering curves; http://kur.web.psi.ch/sans1/SANSSoft/ sasfit.html. (accessed Oct 01, 2012).

(27) Goldmints, I.; von Gottberg, F. K.; Smith, K. A.; Hatton, T. A. Small-Angle Neutron Scattering Study of PEO-PPO-PEO Micelle Structure in the Unimer-to-Micelle Transition Region. Langmuir 1997, $13,3659-3664$. 
(28) Batsberg, W.; Ndoni, S.; Trandum, C.; Hvidt, S. Effects of Poloxamer Inhomogeneities on Micellization in Water. Macromolecules 2004, 37, 2965-2971.

(29) Loh, W. Block copolymer micelles. In Encyclopedia of Surface and Colloid Science, Hubbard, T. A., Ed.; Marcel Dekker: New York; pp 802813. 